\title{
Calcium-Dependent Depolarizations Originating in Lizard Motor Nerve Terminals
}

\author{
Kiichiro Morita and Ellen F. Barrett \\ Department of Physiology and Biophysics, University of Miami School of Medicine, Miami, Florida 33101
}

Action potentials and afterpotentials were recorded via a microelectrode inserted into motor axons innervating the lizard ceratomandibularis muscle. Intra-axonal injection of Lucifer yellow dye indicated that these axons innervate multiple (at least 6-25) motor terminals. In $10 \mathrm{~mm}$ tetraethylammonium (TEA), the action potential was followed by a sequence of afterpotentials, whose amplitude and duration increased with increasing proximity to motor nerve terminals. In axons impaled within $1 \mathrm{~mm}$ of their most distal terminals, these afterpotentials included a depolarizing plateau (mean amplitude and duration: $32 \mathrm{mV}, 24 \mathrm{msec}$ ) and a subsequent smaller depolarization that decayed over a time course of several hundred milliseconds. These depolarizing afterpotentials were $\mathrm{Ca}$ dependent: They increased with increasing bath [Ca] and were abolished by low [Ca]-high [Mg] solutions, by $\omega$-conotoxin (GVIA, $1 \mu \mathrm{M}$ ), by addition of Cd (1 $\mu \mathrm{M})$ or $\mathrm{Mn}(0.3-1 \mathrm{mM})$ to the bath, and by selective perfusion of $\mathrm{Cd}$ over the terminal region. In [Ca]-free solutions the afterpotentials were restored by selective perfusion of Ca over the terminal region but not by $\mathrm{Ca}$ applied to the more proximal nerve trunk. When $\mathrm{Na}$ influx was eliminated by 1-10 $\mu \mathrm{M}$ tetrodotoxin or by substitution of TEA for bath $\mathrm{Na}$, passage of depolarizing current into the axon evoked prolonged depolarizing afterpotentials that were blocked by Mn. Bay K $8644(0.1-1 \mu \mathrm{M})$, a dihydropyridine that prolongs the opening of certain calcium channels, enhanced mainly the slower component of the depolarizing afterpotential. Nimodipine (0.1-1 $\mu \mathrm{M})$, a dihydropyridine that favors the closed state of some calcium channels, shortened the plateau phase of the depolarizing afterpotential. Another antagonist dihydropyridine, nitrendipine $(0.1-1 \mu \mathrm{M})$, had little or no effect on the depolarizing afterpotential but did antagonize the actions of Bay $K \mathbf{8 6 4 4}$. These results suggest that the intraaxonally recorded $\mathrm{Ca}$-dependent afterpotentials are caused by electrotonic spread of depolarizations produced by calcium influx into that axon's terminals and that some motor nerve terminal calcium channels are sensitive to certain dihydropyridines.

\footnotetext{
Received Oct. 11, 1988; revised Mar. 10, 1989; accepted Mar. 11, 1989.

This work was supported by grants NS 12404 and GM 30377 from the National Institutes of Health. We thank Ellen McGlade-McCulloh and John Barrett for reading preliminary versions of the manuscript, and John Barrett for injecting dye.

Correspondence should be addressed to Ellen F. Barrett, Department of Physiology and Biophysics, University of Miami School of Medicine, PO Box 016430, Miami, FL 33101.

- Present address: Department of Autonomic Physiology, Medical Research Institute, Tokyo Medical and Dental University, Tokyo, 101 Japan.

Copyright (c) 1989 Society for Neuroscience $0270-6474 / 89 / 093359-11 \$ 02.00 / 0$
}

Much of our knowledge concerning mechanisms of chemical synaptic transmission has been derived from experiments using the vertebrate neuromuscular junction (reviewed in Katz, 1969; Rahamimoff et al., 1978; Silinsky, 1985). However, the small size of motor nerve terminals has hindered attempts to study the transmembrane currents that influence their membrane potential, calcium influx, and transmitter release. Recordings of end-plate potentials and extracellular recordings from pipettes situated adjacent to motor nerve terminals or in the subperineurial sheath near terminals indicate that these terminals are enriched in calcium channels and that calcium currents can become regenerative in the presence of potassium channel blocking drugs (Katz and Miledi, 1969b; Brigant and Mallart, 1982; Gundersen et al., 1982; Mallart, 1984, 1986; Penner and Dreyer, 1986; Hamilton and Smith, 1987; Lindgren and Moore, 1989). We describe here intra-axonal recordings of Ca-dependent potentials originating in the terminals of single lizard motor axons. The large, $\mathrm{Ca}$-dependent potentials recorded in the presence of tetraethylammonium (TEA) exhibit pharmacological sensitivities characteristic of L-type channels and persist for hundreds of milliseconds following the action potential. Some of these results have been presented in abstract form (Barrett and Morita, 1987).

\section{Materials and Methods}

Preparation and solutions. Experiments were performed on the ceratomandibularis nerve-muscle preparation dissected from small lizards (Anolis sagrei). The standard perfusing saline contained (in $\mathrm{mM}$ ): $\mathrm{NaCl}$, $157 ; \mathrm{KCl}, 4 ; \mathrm{CaCl}_{2}, 2 ; \mathrm{MgCl}_{2}, 2$; glucose, 5 ; and PIPES buffer, 1 . Bath $\mathrm{pH}$ was adjusted to 7.0-7.4 and monitored colorimetrically using the indicator dye phenol red. In addition, most solutions contained $10 \mathrm{~mm}$ tetraethylammonium bromide (TEA, see below). All experiments were carried out at room temperature $\left(20-25^{\circ} \mathrm{C}\right)$. Superfusing solutions were changed (within $1 \mathrm{~min}$ ) using a Gilson perfusion pump. When the composition of the bathing solution was altered, total osmolarity was maintained in the range of 300-310 mOsm by adjusting the concentration of $\mathrm{NaCl}$. In some cases, ions or drugs were applied to a localized region by pressure ejection (General Valve) using a micropipette with a tip diameter of $10-20 \mu \mathrm{m}$. The micropipette was moved into position just prior to drug application. Pressures of $760-1520 \mathrm{~mm} \mathrm{Hg}$ were applied in $1-3$ pulses $50-100 \mathrm{msec}$ in duration, and applied drugs were removed with a nearby suction pipette.

Axonal action potentials were usually evoked by applying suprathreshold depolarizing pulses $(20 \mu \mathrm{sec}-1 \mathrm{msec}$ at $\leq 0.1 \mathrm{~Hz}$ ) to the nerve trunk via a suction electrode. Muscle contractions were prevented by blocking the end-plate ACh receptors with carbachol (30-100 $\mu \mathrm{M}$ ), which in this concentration range does not alter recorded intra-axonal potentials.

Most reagents were obtained from Sigma. TEA came from Aldrich. $\omega$-Conotoxin (GVIA) was kindly provided by Dr. Baldomero Olivera of the University of Utah, and the dihydropyridines nitrendipine and Bay K 8644 by Dr. Alexander Scriabine of Miles Laboratories. Dihydropyridines were added from fresh stock solutions (100 $\mu \mathrm{M}-1 \mathrm{mM}$ in 

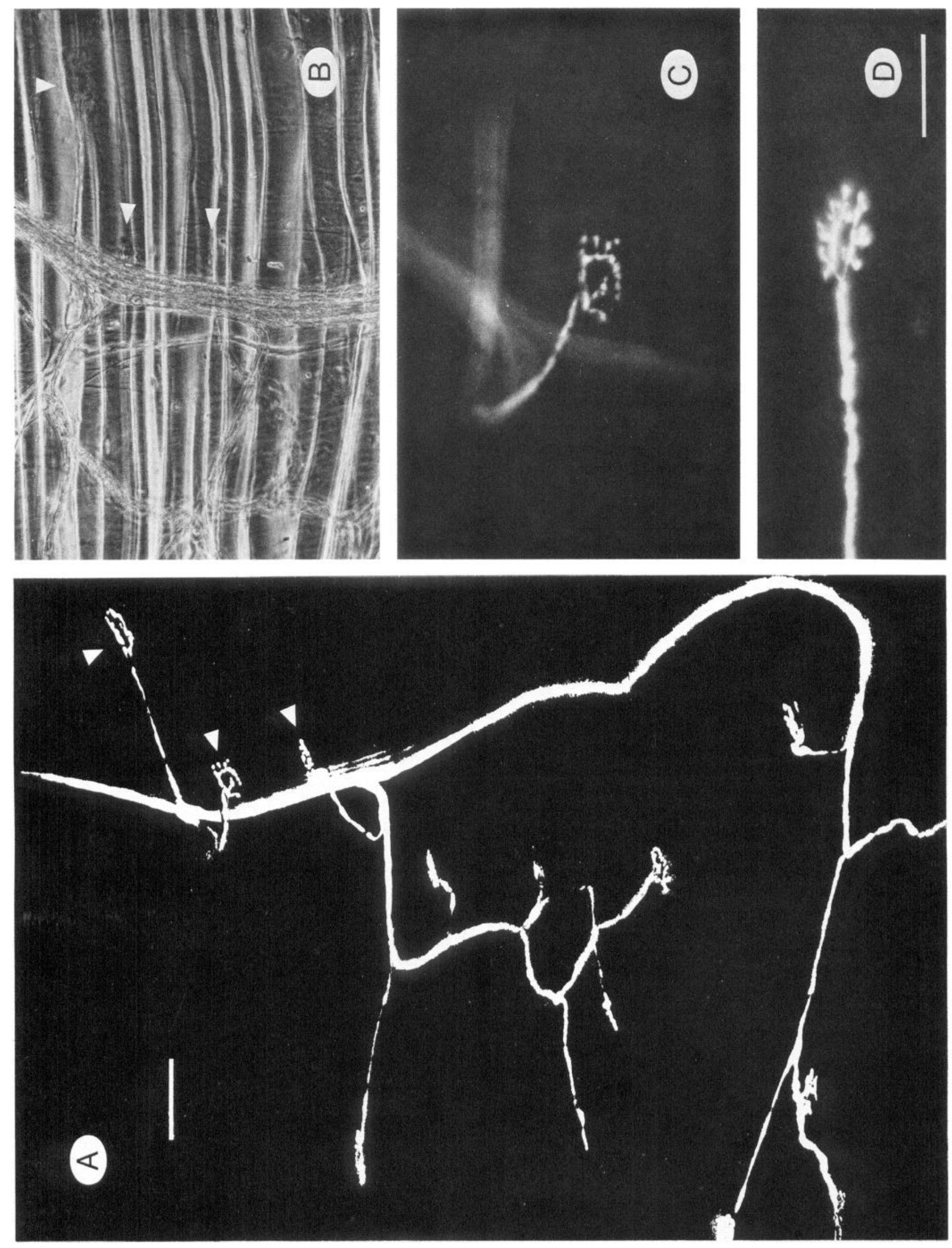


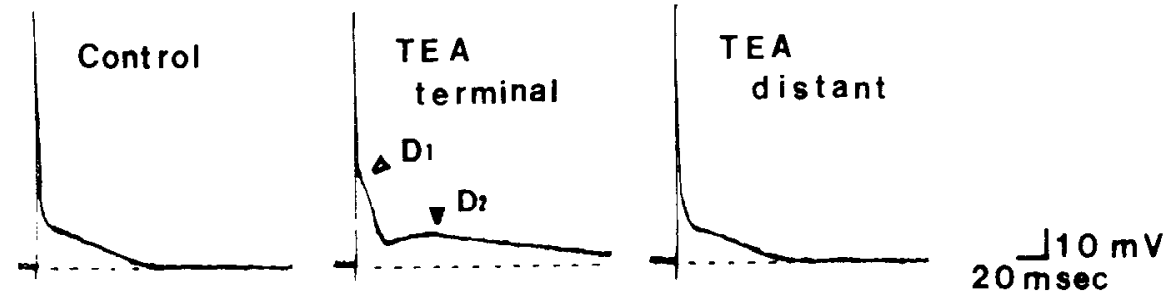

B

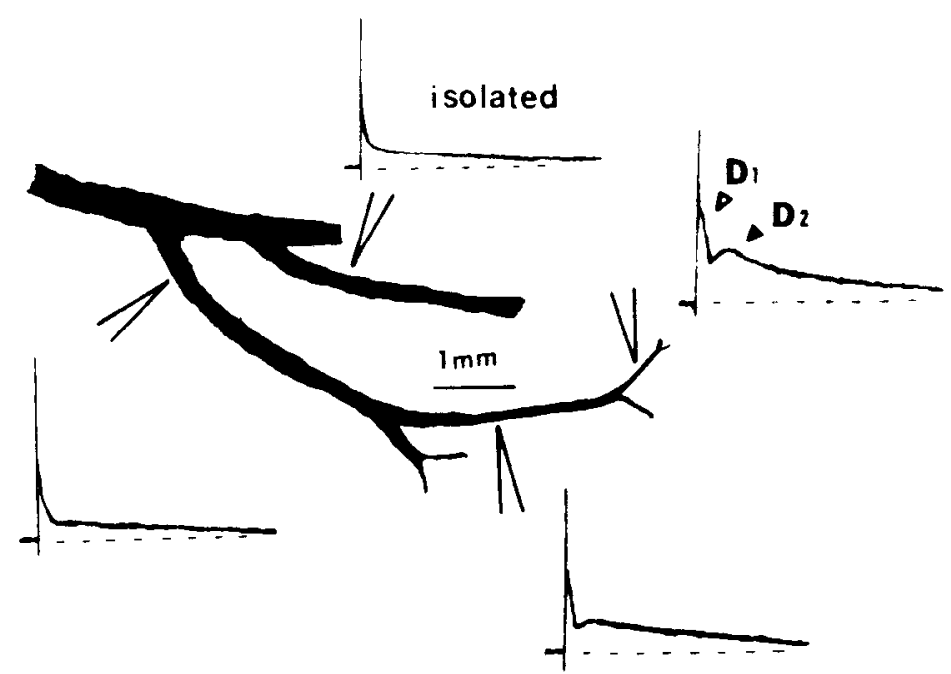

Figure 2. 1, Effect of TEA on the depolarizing afterpotential in an axon impaled near its motor terminals. Left trace recorded in the absence of TEA; middle and right traces, 2-3 sec after localized pressure ejection of TEA onto either the terminal region or the nerve trunk 2-3 mm distant from terminals, respectively. Afterpotential components labeled D1 and D2 were prominent only when TEA was present around the terminals. The ejecting pipette was filled with 100 mM TEA. Resting potential, $-73 \mathrm{mV}$. $B$, The thick line shows a typical nerve trunk branching pattern. Insets show action potentials and depolarizing afterpotentials recorded at the 4 indicated recording siles in axons bathed in 10 mм TEA. Each recording came from a separate axon but was typical of recordings made at that location in 5-10 other axons. The D1 and D2 components of the depolarizing afterpotential were largest at the more distal recording sites. The trace labeled "isolated" was recorded in an axon cut off from its terminals; here, the depolarizing afterpotential decayed monotonically, reflecting passive discharge of the internodal axolemmal capacitance (Barrett and Barrett, 1982). In this and subsequent figures, dashed lines indicate baselines. Scale bars: $10 \mathrm{mV}, 20 \mathrm{msec}$ in $A ; 1 \mathrm{~mm}, 20 \mathrm{mV}, 50 \mathrm{msec}$ in $B$. ethanol) and were always shielded from light; control solutions contained equivalent ethanol concentrations.

Intra-axonal recordings. Intra-axonal recordings were made with glass micropipettes filled with $0.5 \mathrm{~m}$ potassium sulfate, with a tip resistance in the range of 30-100 M 2 , using techniques similar to those described in Barrett et al. (1988). Low-resistance pipettes were selected for those experiments in which the recording pipette was also used to pass current into the axon via a bridge circuit. The micropipette was inserted into the internode of a visualized axon using a piezoelectric device (Frederick Haer) at varying distances (up to $10 \mathrm{~mm}$ ) from the most distal motor terminals. (In some cases the location of motor terminals was verified by recording end-plate potentials in an underlying muscle fiber.) The intra-axonal recordings illustrated in Figures 3-10 were made within 1 $\mathrm{mm}$ of the most distal motor terminals. In a few experiments, axons were mechanically separated from their motor terminals.

Recorded action potentials and afterpotentials were amplified using a Dagan 8800 amplifier, displayed on an oscilloscope (Tektronix, R5103n), and recorded on tape (Hewlett Packard 3960). Changes in resting potentials and the slower afterpotentials were monitored using a Gould $2200 \mathrm{~S}$ chart recorder. The final resting membrane potential was determined as the microelectrode was withdrawn from the axon. Changes in tip potential resulting from changes in the composition of the bathing solution were controlled for by recording differentially between the microelectrode in the axon and a matched microelectrode in the bath. Average measurements are reported as mean $\pm \mathrm{SEM}$.
TEA was used to enhance the recorded depolarizing afterpotentials. As discussed in Barrett et al. (1988), TEA blocks a variety of K channels, including $\mathrm{K}$ channels open in the resting axon, thus increasing the axonal space constant and reducing the effective electrical distance between the motor nerve terminals and the intra-axonal recording site. Most axons were exposed to TEA for at least $1 \mathrm{hr}$ prior to recording because the TEA-induced reduction of axonal input conductance reaches a steadystate slowly, probably due to delayed diffusion of TEA to sites on the internodal axolemma (Barrett et al., 1988). TEA depolarized the axonal resting potential (by about $12 \mathrm{mV}$ in $10 \mathrm{~mm}$ TEA), but all axons included in this study had resting potentials of $-50 \mathrm{mV}$ or better, and action potential amplitudes of at least $50 \mathrm{mV}$. The mean resting potential in TEA was $63 \pm 0.8 \mathrm{mV}$ SE $(n=25)$. Preparations in TEA remained electrically healthy for 5-6 hr. Some solutions also contained $0.1-1 \mathrm{mM}$ 4-aminopyridine.

Intra-axonal dye injection. In separate experiments, single axons were impaled near their terminals with a microelectrode filled with $10 \mathrm{mg} /$ $\mathrm{ml}$ Lucifer yellow. Dye was iontophoresed into the axon with a $0.3-0.5$ nA hyperpolarizing current for $1-3 \mathrm{hr}$. The preparation was then viewed under a fluorescence microscope and dye-filled motor terminals (Fig. 1) were counted. These counts were probably underestimates: terminals may have been missed due to inadequate penetration of dye into processes distant from the injection site, and the opacity of occasional damaged muscle fibers obscured the fluorescence of terminals ending on them. Staining of motor terminals was specific for the injected axon.

Figure 1. A, Montage of fluorescence micrographs showing a portion of the terminal field of a lizard motor axon injected for 2 hr with Lucifer yellow dye. This axon had 23 distinct dye-filled terminals, 3 of which are indicated by arrowheads. $B$, Phase-contrast micrograph of the upperright region of $A$, which includes the site of axonal impalement. Arrowheads indicate the positions of the 3 motor terminals labeled in $A$. $C$, Enlargement of the middle dye-filled terminal labeled in $A$. $D$, Dye-filled terminal from another injected axon. Both $C$ and $D$ show numerous varicosities along the terminal branches. Scale bar in $A$ indicates $100 \mu \mathrm{m}$ in $A$ and $B$ and $50 \mu \mathrm{m}$ in $C$; bar in $D$ indicates $50 \mu \mathrm{m}$. 
A
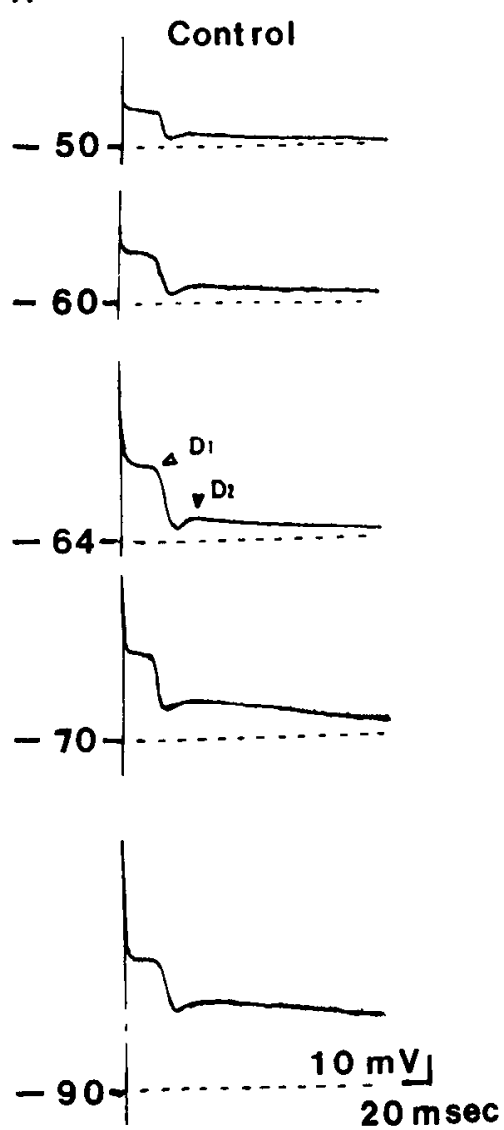

Figure 3. Changes in afterpotential configuration in a motor axon during passage of steady currents through the intra-axonal recording electrode before $(A)$ and 5-10 min after $(B)$ addition of $1 \mathrm{~mm} \mathrm{Mn}$ to the bath. TEA, $10 \mathrm{~mm}$, was present throughout. The number at the left of each trace indicates the baseline membrane potential measured at the recording site; changes in membrane potential at the terminals were smaller. Mn blocked the D1 and D2 components of the depolarizing afterpotential, leaving a passive component. Changes in this passive component dominate the recorded response to injected current, and these changes in axonal passive properties would alter the electrotonic distance between the intra-axonal recording site and the terminals. The Ca-dependent component of the depolarizing afterpotential can be estimated by subtracting the afterpotential recorded in Mn from that recorded at the same potential in the absence of $\mathrm{Mn}$. At the resting potential $(-64 \mathrm{mV})$, this subtraction procedure yielded a D1 amplitude of $22 \mathrm{mV}$, a D1 duration of $30 \mathrm{msec}$, and a peak D2 amplitude of 4 $\mathrm{mV}$. Amplitudes measured by this subtraction procedure are unlikely to be exactly proportional to terminal Ca currents because of the variable electrotonic distance between the recording site and the various terminals, and because these terminals also have $\mathrm{Ca}$-dependent $\mathrm{K}$ conductances that are inhibited by Mn (Morita and Barrett, 1988). Scale bar: $10 \mathrm{mV}, 20 \mathrm{msec}$.

Addition of dye to the bath (either intentionally or as a result of slippage of the dye-injecting electrode) faintly stained all myelinated portions of the nerve trunk but never stained motor terminals.

\section{Results}

Figure 1 shows fluorescence $(A)$ and phase-contrast $(B)$ micrographs of portions of the terminal field of a lizard motor axon injected with Lucifer yellow dye via an intra-axonal micropipette. Axons innervated terminals along several millimeters of the muscle surface; in 5 experiments the injected axon gave rise to (at least) $6,11,22,23$, and 25 terminals. This result suggests that the intra-axonally recorded potentials described below represent a summation of potentials arising in the myelinated axon and its multiple motor terminals. The detailed morphology of injected terminals (Fig. 1,C,D) resembled that of Walrond and Reese's (1985) zinc iodide-stained motor terminals on lizard intercostal muscle, consistent with their suggestion that the interconnected boutons are varicosities along the terminal branches of a single axon.

\section{Depolarizing afterpotentials originating in motor nerve terminals}

Action potentials evoked in lizard motor axons are followed by a prolonged, monotonically decaying depolarizing afterpotential (left trace of Fig. $2 A$; lower traces of Fig. $3 B$ ). Previous work suggests that this is a passive potential, arising from discharge of the capacitance of the internodal axolemma (Barrett and Barrett, 1982). The configuration of the afterpotential changed dramatically when the terminal regions of the axon were perfused with TEA (middle trace, Fig. $2 A$ ). An early plateau-like depolarization (labeled D1) appeared, followed by a partial repolarization and a subsequent, slowly decaying depolarization (labeled D2). This dramatic change in afterpotential configuration did not occur when TEA application was restricted to the proximal nerve trunk (right trace, Fig. $2 A$ ).

Figure $2 B$ shows further evidence that the D1 and D2 components of the depolarizing afterpotential arose in motor terminals. Here, the entire preparation was bathed in $10 \mathrm{~mm}$ TEA, and action potentials and depolarizing afterpotentials were sampled at the indicated sites. Both D1 and D2 components of the depolarizing afterpotential increased in amplitude as the recording site moved distally along the axon. Sometimes additional action potentials were observed during the D1 plateau. Also, there were occasionally notches on the falling phase of $\mathrm{D} 1$, perhaps reflecting different $\mathrm{D} 1$ durations in the various motor terminals contributing to the recorded potential. Axonal sites remote from terminals, or mechanically isolated from their terminals (upper trace of Fig. $2 B$ ), showed only the monotonically decaying passive depolarizing afterpotential.

D1 and D2 afterpotentials were also seen when the action potential was evoked by injecting depolarizing current through the intra-axonal recording electrode rather than by stimulating the entire nerve trunk (compare left and middle traces in Fig. 4A).

In a sample of 25 axons bathed in $10 \mathrm{~mm}$ TEA and impaled within $1 \mathrm{~mm}$ of their most distal terminals, the Dl afterpotential had a mean peak amplitude of $32 \pm 0.5 \mathrm{mV}$ (mean $\pm \mathrm{SE}$, measured from the resting potential) and declined to $80 \%$ of this peak amplitude an average of $24 \pm 1.2 \mathrm{msec}$ after the falling phase of the action potential. The D2 component reached an average peak amplitude of $14 \pm 0.5 \mathrm{mV}$ at an average latency of $59 \pm 2.1 \mathrm{msec}$ and decayed over a time course of hundreds of msec.

Figure $3 \mathrm{~A}$ shows the changes in the depolarizing afterpotential produced by passing stcady depolarizing or hyperpolarizing currents through the intra-axonal microelectrode. Figure $3 B$ shows records obtained from the same axon after addition of $1 \mathrm{~mm}$ $\mathrm{Mn}$, which blocks D1 and D2, but not the passive component of the depolarizing afterpotential (Barrett and Barrett, 1982; Barrett et al., 1988). In both solutions the depolarizing afterpotentials became larger with membrane hyperpolarization and smaller with membrane depolarization. A similar variation of afterpotential amplitude with membrane potential was noted in 
A
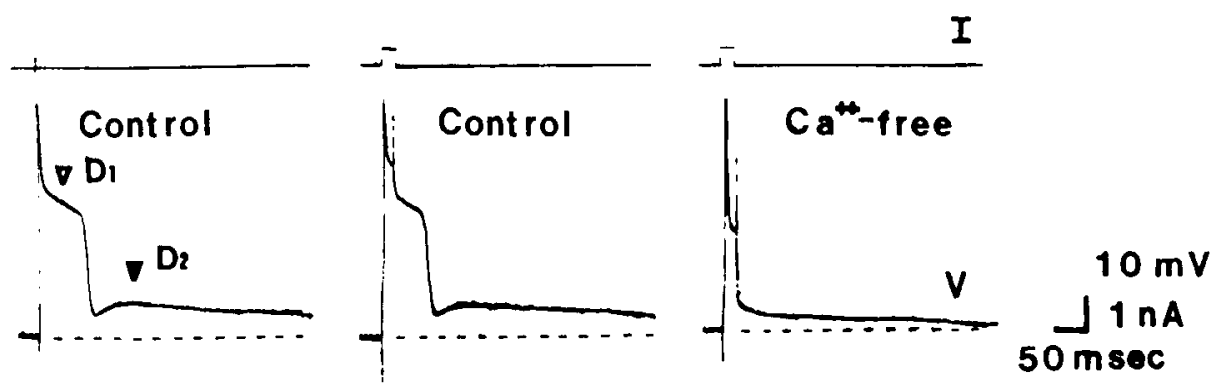

B
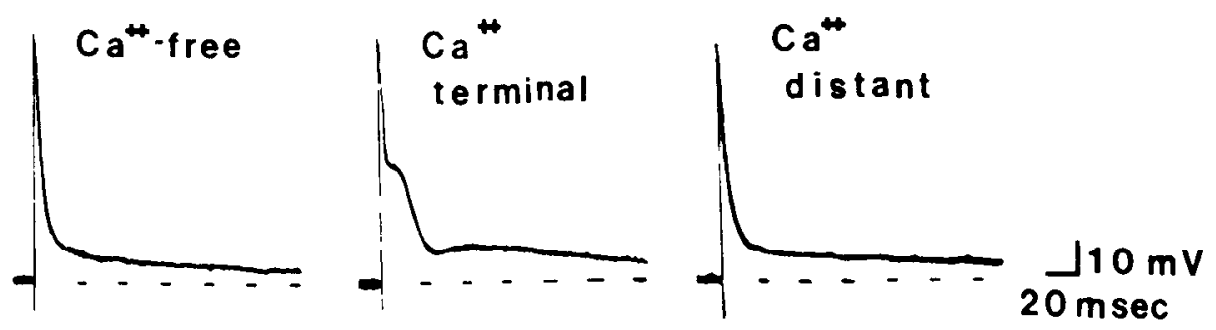

Figure 4. Calcium dependence of D1 and D2 components of the depolarizing afterpotential. $A$, D1 and D2 recorded in $10 \mathrm{~mm}$ TEA and $0.1 \mathrm{~mm}$ 4-aminopyridine were similar whether the action potential was evoked by stimulating the proximal nerve trunk via a suction electrode (left) or by passing depolarizing current into the axon via the recording microelectrode (center). DI and D2 disappeared when Ca was omitted from the bath and $[\mathrm{Mg}]$ was increased from the normal $2 \mathrm{mM}$ to $4 \mathrm{mM}($ right). Lower traces show voltage; upper traces, injected current $(0.5 \mathrm{nA}, 20 \mathrm{msec})$. Low$\mathrm{Ca}$, high-Mg solutions hyperpolarized the resting potential by up to $10 \mathrm{mV}$ (mean, $3.2 \pm 0.8 \mathrm{mV}, n=10$ ) and sometimes produced slight changes in input resistance. However, the disappearance of D1 and D2 was not due to this small hyperpolarization because hyperpolarizing currents applied in $2 \mathrm{mM}$ Ca did not abolish D1 and D2 (Fig. 3A), and depolarizing currents applied in Ca-free solutions did not restore D1 and D2. $B$, D1 and D2 were absent in (nominally) Ca-free medium containing $10 \mathrm{~mm}$ TEA (left); they appeared following localized pressure ejection of Ca over the motor terminals (center) but not when Ca was ejected over the nerve trunk at a site distant from terminals (right). The ejecting pipette contained $50 \mathrm{~mm}$ Ca. Resting potentials: $-62 \mathrm{mV}$ in $A ;-64 \mathrm{mV}$ in $B$. Scale bars: $10 \mathrm{mV}, 1 \mathrm{nA}, 50 \mathrm{msec}$ in $A ; 10 \mathrm{mV}, 20 \mathrm{msec}$ in $B$.

comparisons of records from axons with different resting potentials, sampled at comparable locations (not shown). The records of Figure 3 show that much of the voltage dependence of the afterpotential amplitude was due to the voltage dependence of the passive component. This passive component, generated throughout the myelinated axon, would be expected to be more sensitive to injected current than the terminal-specific D1 and D2 components because the terminals were electrically distant from the intra-axonal site of current injection. Both D1 and D2 afterpotentials were present at a membrane potential of -50 $\mathrm{mV}$, where the passive depolarizing afterpotential was no longer present. The hyperpolarizing afterpotential seen at $-50 \mathrm{mV}$ in Mn may be due to a TEA- and Ca-insensitive $\mathrm{K}$ conductance described in mammalian myelinated axons by Baker et al. (1987) and Gordon et al. (1988).

\section{Terminal-specific depolarizing afterpotentials are Ca-dependent}

The D1 and D2 components of the depolarizing afterpotential were reversibly abolished within $10 \mathrm{~min}$ after perfusing the preparation with a solution containing no added calcium and 2-10 $\mathrm{mm} \mathrm{Mg}$ (right trace, Fig. $4 A$ ). The afterpotential remaining in low [Ca] solutions was usually similar to the passive depolarizing afterpotential described above (Fig. $3 B$ ); in some axons (especially those with resting potentials more depolarized than -60 $\mathrm{mV}$ ), there was instead a brief hyperpolarizing afterpotential (as in top trace of Fig. $3 B$ ). The D1 and D2 afterpotential components reappeared when $\mathrm{Ca}$ was restored to the bath or locally perfused over the terminal region but not when $\mathrm{Ca}$ was locally perfused over the myelinated nerve trunk (Fig. $4 B$ ). Low [Ca], high $[\mathrm{Mg}]$ solutions had little or no effect on the action potential and passive afterpotential recorded in the absence of TEA (not shown).

The amplitude and duration of D1 and D2 increased with increasing bath [Ca]. Figure $5 \mathrm{~A}$ shows typical afterpotentials recorded in one axon as $\mathrm{Ca}$ was gradually added to a (nominally) Ca-free solution, and Figure $5 B$ plots the normalized average changes in the amplitudes of D1 and D2 and the duration of D1 recorded in 3-5 such experiments. Note that D2 became evident at a lower [Ca] $(100 \mu \mathrm{M})$ than D1. D1 reached its maximal amplitude at about $1 \mathrm{~mm} \mathrm{Ca}$; further elevation of bath [Ca] increased the duration of the D1 plateau (Fig. 5, $B-D$ ). In high bath [Ca] repolarization following the D1 plateau was prolonged, making it difficult to measure the amplitude of the slower D2 component. Also, the stimulation rate had to be reduced from the normal $0.1 \mathrm{~Hz}$ to avoid progressive changes in afterpotential configuration. The D1 plateau was also greatly prolonged when bath $\mathrm{Ca}$ was replaced by $2 \mathrm{~mm}$ Ba (K. Morita and E. F. Barrett, unpublished observations).

D1 and D2 were reversibly reduced by locally perfusing the terminals with $\mathrm{Cd}$ but not by similarly perfusing the myelinated nerve trunk (Fig. 6A). D1 and D2 were also reversibly abolished by adding to the bath $1 \mu \mathrm{M} \mathrm{Cd}$ (Fig. $6 B$ ) or $1 \mathrm{mM} \mathrm{Mn,} \mathrm{Ni,} \mathrm{or}$ Co (Fig. 3B; Barrett et al., 1988; Morita and Barrett, unpublished observations). This inhibition of D1 and D2 could be partially reversed by elevating bath [Ca] (not shown). 

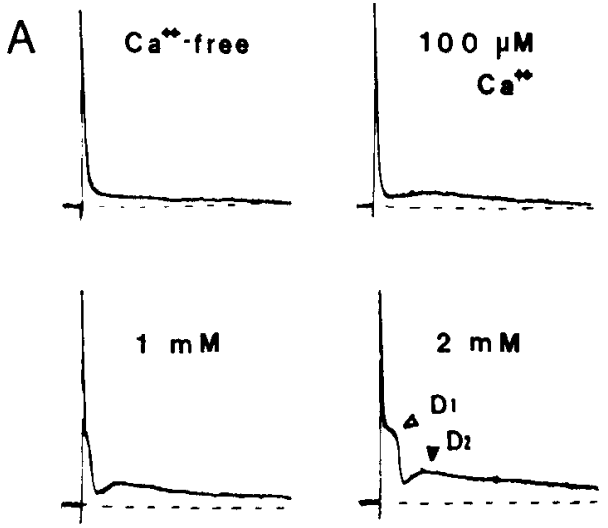

$110 \mathrm{mV}$

C

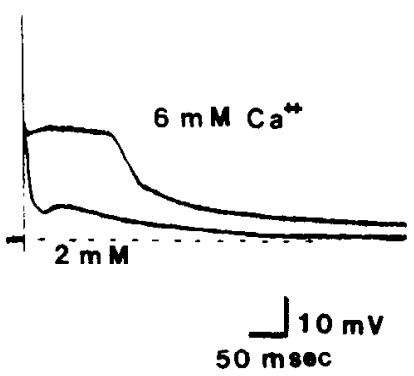

B
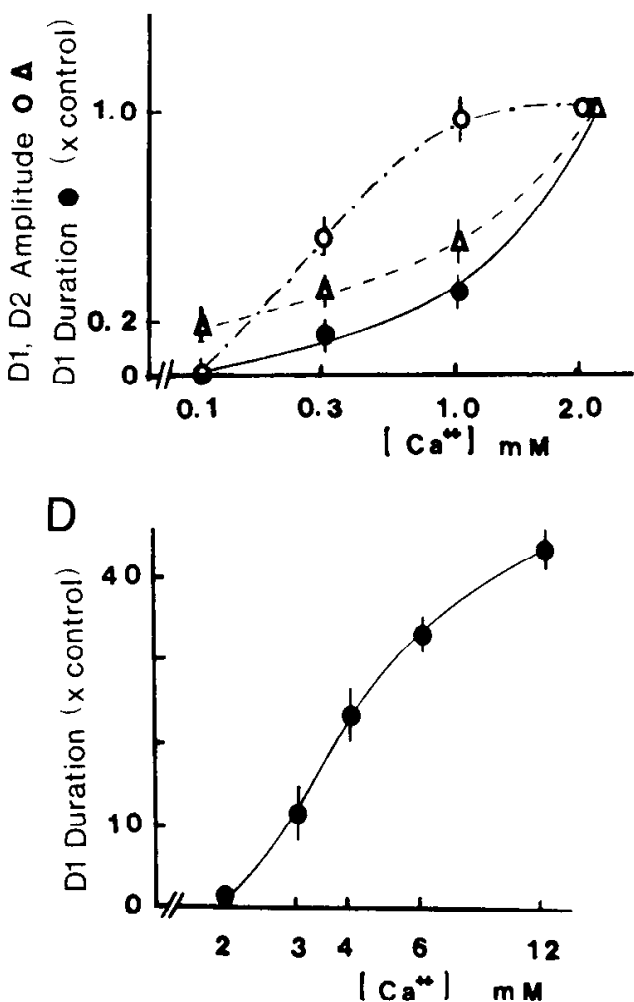

Figure 5. Dependence of D1 and D2 components of the depolarizing afterpotential on bath [Ca] in the presence of $10 \mathrm{~mm}$ TEA. $A$, Afterpotentials recorded in one axon in a nominally Ca-free medium, and $5 \mathrm{~min}$ after exposure to $100 \mu \mathrm{M}, 1 \mathrm{mM}$, and $2 \mathrm{mM} \mathrm{Ca}(2 \mathrm{mM} \mathrm{Mg}$ present throughout). Between each exposure to $\mathrm{Ca}$ the axon was perfused 5-7 min with a $\mathrm{Ca}$-free solution. Resting potential $-63 \mathrm{mV}$. $B$, Increases in peak Dl amplitude (open circles), D1 duration (filled circles), and peak D2 amplitude (triangles) with bath [Ca] in the range $100 \mu \mathrm{M}-2 \mathrm{~mm}$ (logarithmic scale). D1 duration was measured as the interval between the falling phase of the action potential and the time at which D1 had declined to $80 \%$ of its peak amplitude. D1 and D2 amplitudes were measured as the difference between the recorded peak amplitudes and the (passive) afterpotential recorded at the corresponding time in (nominally) Ca-free solution. Amplitudes and duration are plotted relative to values measured in $2 \mathrm{~mm}$ Ca [DI amplitude, $26 \pm 2.4 \mathrm{mV}$ (mcan $\pm \mathrm{SEM}$ ); D2 amplitude, $12 \pm 1.4 \mathrm{mV}$; D1 duration, $26 \perp 3.3 \mathrm{msec}, n=7]$. Bars indicate SEM from measurements in 3-5 axons. $C$, Superimposed traces of afterpotentials recorded in another axon in 2 and $6 \mathrm{~mm} \mathrm{Ca}$. Elevation of bath [Ca] tended to hyperpolarize axons slightly $(2-3 \mathrm{mV})$, with no detectable change in input resistance. Resting potential, $-70 \mathrm{mV}$. $D$, Increase in mean duration of D1 afterpotential with bath [Ca] in the range 2-12 $\mathrm{mM}$ (logarithmic scale). Duration is plotted relative to value measured in $2 \mathrm{mM} \mathrm{Ca}$. Bars indicate SEM from measurements in 4-6 axons. Lines in $B$ and $D$ were drawn by eye. Scale bars: $10 \mathrm{mV}$ in $A, C ; 20 \mathrm{msec}$ in $A, 50 \mathrm{msec}$ in $C$.

To determine whether the terminal-specific, Ca-dependent potentials could be elicited simply by depolarization, without the $\mathrm{Na}$ influx associated with the action potential, we passed depolarizing current into axons bathed in 1-10 $\mu \mathrm{M}$ tetrodotoxin (TTX) in addition to $10 \mathrm{~mm}$ TEA. In axons near their terminals, but not in remote or isolated axons, this applied depolarization evoked a regenerative depolarization during the current pulse and a slowly decaying depolarizing afterpotential, both of which were reduced or abolished by focal application of $\mathrm{Cd}$ or by addition of $1 \mathrm{~mm}$ Mn to the bath (Fig. 7). Similar Mn-blockable potentials were evoked in axons depolarized in a solution in which TEA bromide was substituted for $\mathrm{NaCl}$ (Fig. 8).

\section{Effects of organic Ca channel-blocking agents}

$\omega$-Conotoxin (GVIA), which blocks several types of Ca channels, including those that mediate transmission at the frog neuromuscular junction (Kerr and Yoshikami, 1984; Feldman et al., 1987; McCleskey et al., 1987), blocked both D1 and D2 components of the depolarizing afterpotential in a dose-dependent manner. Inhibition was first evident at $30 \mathrm{nM}$, with complete block at $1 \mu \mathrm{M}$ (Fig. 9). The inhibition produced by $1 \mu \mathrm{M}$ conotoxin was not reversible following toxin washout. This result agrees with Lindgren and Moore's (1989) results on extracellularly recorded $\mathrm{Ca}$-dependent currents at lizard motor nerve terminals. Interestingly, $\omega$-conotoxin does not block transmission or presynaptic $\mathrm{Ca}$ channels at the mouse neuromuscular junction (Anderson and Harvey, 1987; Sano et al., 1987).

Certain types of $\mathrm{Ca}$ channel are altered by dihydropyridine drugs, which are classified as agonists (e.g., Bay K 8644) or antagonists (e.g., nitrendipine, nimodipine), depending on whether they favor the prolonged open or the inactivated mode of these Ca channels (Hess et al., 1984). Bay K 8644 (0.1-0.3 $\mu \mathrm{M})$ reversibly increased the amplitude of the D2 component of the depolarizing afterpotential (Fig. 10A). At higher concentration (1 $\mu \mathrm{M})$, Bay K 8644 sometimes produced a slight prolongation of the D1 component (not shown). These effects of Bay $\mathrm{K} 8644$ were antagonized by nitrendipine $(0.1-1 \mu \mathrm{M})$, which by itself had little or no effect on D1 and D2 (not shown). Nimodipine $(0.1 \mu \mathrm{M})$ reversibly shortened the D1 plateau by about $25 \%$ (Fig. 10B) but did not alter D2. At higher concentration ( $1 \mu \mathrm{M})$, nimodipine shortened D1 by aboul $60 \%$ and reduced D2 amplitude by about 30\% (not shown). All reported effects 

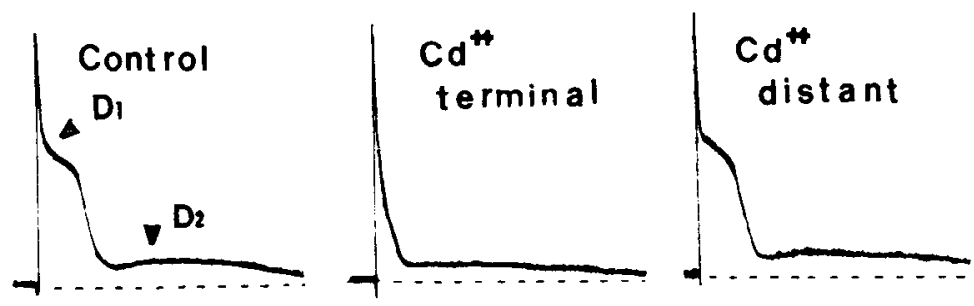

\section{$-10 \mathrm{mV}$ $20 \mathrm{msec}$}

B

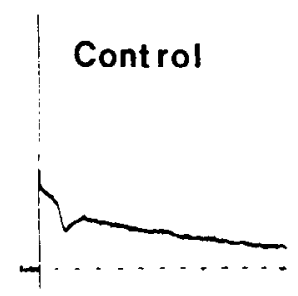

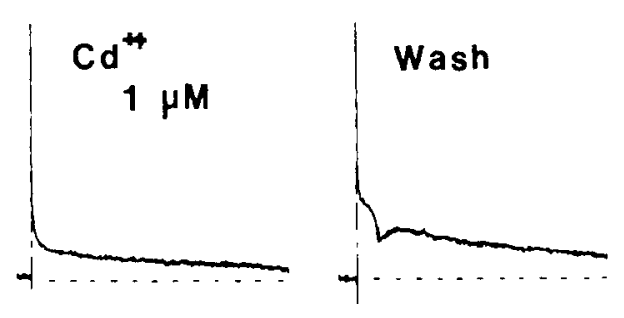

Figure 6. Inhibition of D1 and D2 components of depolarizing afterpotential by cadmium. $A, \mathrm{D} 1$ and D2 (left trace) were reduced when $\mathrm{Cd}$ was locally ejected over the terminal region (middle trace) but not when $\mathrm{Cd}$ was ejected over the nerve trunk $2-3 \mathrm{~mm}$ distant from terminals (right trace). The perfusion pipette contained $100 \mu \mathrm{M} \mathrm{Cd}$. Similar results were obtained in 3 additional experiments. $B, \mathrm{D} 1$ and $\mathrm{D} 2$ were reversibly abolished by addition of $1 \mu \mathrm{M} \mathrm{Cd}$ to the bathing solution. TEA, $10 \mathrm{mM}$, was present throughout $A$ and $110 \mathrm{mV} B$. Resting potential, $-62 \mathrm{mV}$ in $A$, -68 $20 \mathrm{msec} \quad$ in $A$ and $B$. of dihydropyridines were observed in 2 or more axons. The differential effects of nimodipine and nitrendipine might be related to the observation that at low concentrations nitrendipine can act as a partial agonist for L-type calcium channels (Hess et al., 1984).

\section{Discussion}

\section{Origin of D1 and D2 components of the depolarizing} afterpotential

Many experimental observations indicate that the intra-axonally recorded, Ca-dependent potentials illustrated here originate mainly in (or near) motor nerve terminals. These potentials appear and increase in amplitude as the intra-axonal recording site approaches the terminal region (Fig. $2 B$ ). Also, the potentials appear in a Ca-free solution following selective pcrfusion of thc terminal region with $\mathrm{Ca}$ (Fig. $4 B$ ) and in a TEA-free solution following perfusion of the terminal region with TEA (Fig. $2 A$ ). The potentials are blocked by perfusion of the terminal region with Cd (Fig. 6A). Because each motor axon gives rise to multiple motor terminals (Fig. $1 A$ ) and because these axons have a long (several millimeters) space constant for slow events (Barrett and Barrett, 1982), it is likely that the recorded Ca-dependent afterpotentials reflect the summed activity of multiple terminals.

In the presence of $10 \mathrm{mM}$ TEA the Ca-dependent afterpoten-
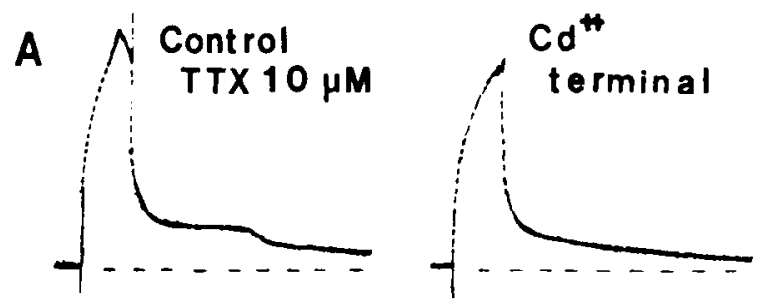

Figure 7. Potentials evoked by injecting depolarizing current pulses (2$3 \mathrm{nA}, 20 \mathrm{msec}$ ) into an axon bathed in $10 \mu \mathrm{M}$ TTX, $10 \mathrm{~mm}$ TEA, and $0.1 \mathrm{~mm}$ 4-aminopyridine. Portions of both the depolarization evoked during the current pulse and the depolarizing afterpotential following the pulse were reversibly blocked by locally perfusing the terminal region with $\mathrm{Cd}(A)$ or by adding $1 \mathrm{~mm} \mathrm{Mn}$ to the bath $(B)$. This record came from the same recording site as Figure $4 A$. The D1 and D2 afterpotentials following the action potential in Figure $4 A$ lasted longer than the Ca-dependent depolarizations recorded here in TTX, probably because the conducted action potential activated $\mathrm{Ca}$ currents in all terminals, whereas the passive, electronically conducted depolarization in TTX activated Ca currents only in those terminals nearest the electrode. The reduced overall depolarizing currents in TTX would cause those Ca-dependent depolarizations that did occur to terminate more quickly. Scale bar: $20 \mathrm{mV}, 20 \mathrm{msec}$. 
Figure 8. Left column shows potentials evoked by injecting depolarizing current pulses into an axon bathed for $30 \mathrm{~min}$ in Na-free solution (TEA substituted for bath $\mathrm{Na}$ ); middle column was recorded during addition of $1 \mathrm{~mm}$ $\mathrm{Mn}$ to this solution; right column, 10 min after washout of Mn. In each column, the upper trace shows injected current ( $2 \mathrm{nA}, 20 \mathrm{msec}$ ), and the middle and lower traces show recorded voltage (greater amplification and slower time sweep in lower trace). Mn reversibly blocks a portion of the depolarization recorded during the current pulse and most of the afterpotential. Resting potential, $-39 \mathrm{mV}$ in Na-free solution, $-44 \mathrm{mV}$ during exposure to $\mathrm{Mn}$. Scale bars: upper traces, $5 \mathrm{nA}, 20 \mathrm{msec}$; middle traces, $10 \mathrm{mV}, 20 \mathrm{msec}$; lower traces, $5 \mathrm{mV}, 2 \mathrm{sec}$. Stimulation frequency, 0.01 $\mathrm{Hz}$.

tial labeled D1 reached a maximal plateau amplitude at a bath [Ca] of about $1 \mathrm{~mm}$. Higher bath [Ca] increased the duration of the D1 plateau (Fig. 5). This behavior suggests that the D1 afterpotential results from $\mathrm{Ca}$-dependent action potentials in the motor terminals, propagated electrotonically back to the intra-axonal recording site. Evidence for $\mathrm{Ca}$ influx into motor terminals has also been obtained using extracellular electrodes placed near a terminal or within distal regions of the perineurial sheath of mouse, rat, and lizard motor axons bathed in TEA and 3,4-diaminopyridine (Brigant and Mallart, 1982; Gundersen et al., 1982; Mallart, 1984, 1986; Hamilton and Smith, 1987; Lindgren and Moore, 1989). Regenerative Ca spikes have been recorded in many other cell membranes treated with TEA (and often TTX), including the somatic membranes of frog and rat motoneurons (Barrett and Barrett, 1976; Alvarez-Leefmans and Miledi, 1980; Walton and Fulton, 1986; Harada and Takahashi, 1983), and various presynaptic nerve terminal membranes (Katz and Miledi, 1969a; Ross and Stuart, 1978; Obaid et al., 1985).

The D2 component of the depolarizing afterpotential also appears to be mediated, at least in part, by Ca influx. D2 probably includes a passive component attributable to discharge of

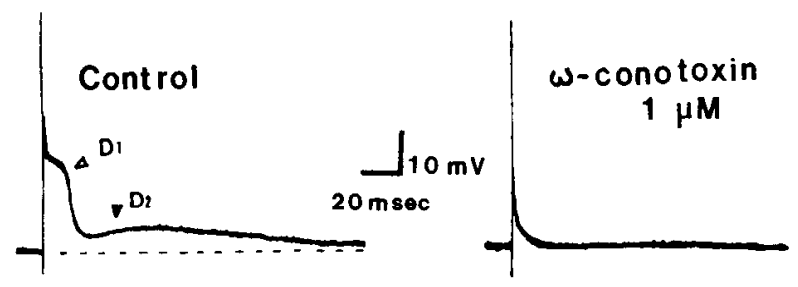

Figure 9. Block of D1 and D2 components of depolarizing afterpotential by $\omega$-conotoxin (GVIA). Afterpotentials before (left) and after (right) exposure to $1 \mu \mathrm{M}$ conotoxin. TEA, $10 \mathrm{~mm}$, present throughout. Resting potential, $-64 \mathrm{mV}$. Scale bars: $10 \mathrm{mV}, 20 \mathrm{msec}$. the axonal-terminal membrane capacitance following the large D1 depolarization, but this passive component cannot account for all D2 because the time course of D2 decay was different from that of the passive depolarizing afterpotential recorded in the presence of $\mathrm{Mn}, \mathrm{Cd}$, or $\omega$-conotoxin (Figs. 3, 6, 9). Also, D2 was present even in axons too depolarized to show a passive depolarizing afterpotential (top trace, Fig. $3 A$ ), and the time course of $\mathrm{D} 2$ decay became slower with depolarization, whereas that of the passive response became faster (Fig. 6 in Barrett et al., 1988). The current mediating the active component of D2 is more likely to be $\mathrm{Ca}$ influx than $\mathrm{Na}$ influx because application of brief depolarizing pulses to axons bathed in TTX or in Nafree solution evoked slow, D2-like depolarizations which were blocked by Mn (Figs. 7, 8).

One possibility is that the recorded D2 potential might be due simply to DI-like Ca spikes in nerve terminals very distant from the recording site, reduced and slowed by electrotonic conduction. However, the finding that Bay K 8644 enhanced D2 but not D1, whereas nimodipine inhibited D1 more than D2 (Fig. 10) makes it seem more likely that D2 reflects a real, but smaller, second depolarization of each terminal, perhaps the beginnings of the oscillations in membrane potential seen at higher TEA concentrations (Morita and Barrett, unpublished observations).

Fast and slow components of motor terminal Ca currents have also been noted in recordings from electrodes inserted into the pcrincurial sheath of mouse motor nerves bathed in TEA and aminopyridines (Mallart, 1985; Penner and Dreyer, 1986). Slow components lasting hundreds of milliseconds in $2 \mathrm{mM}$ Ca have not yet been noted in recordings from electrodes placed next to motor terminals, perhaps due to the small magnitude of the slow inward currents. Another possibility is that the slow currents are reduced by the repetitive stimulation usually used to sum the small extraterminally recorded currents. Mallart (1985) and Penner and Dreyer (1986), and our own observations, in- 
A
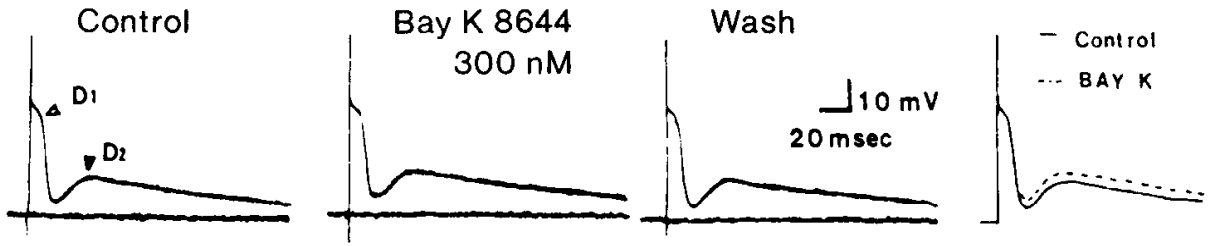

B

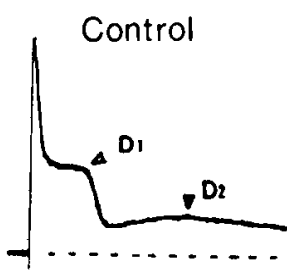

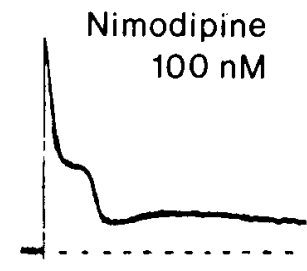

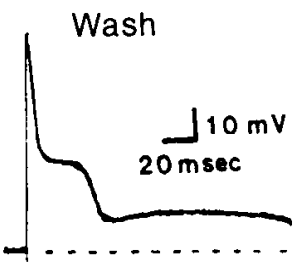

Figure 10. Effects of dihydropyridine agonist Bay K 8644 and antagonist nimodipine on D1 and D2 components of depolarizing afterpotential. Traces (from left to right) show afterpotentials before, during exposure to, and following washout of, Bay $\mathrm{K} 8644(A, 300 \mathrm{nM})$ or nimodipine $(B, 100 \mathrm{nM})$. Insets at far right superimpose afterpotentials before and during exposure to the drug, showing that $300 \mathrm{nM}$ Bay K 8644 selectively increased the D2 component, whereas $100 \mathrm{~nm}$ nimodipine selectively depressed the D1 component. Bay K 8644 depolarized the axonal resting potential by a few millivolts; neither drug altered the axonal passive response to injected current. TEA, $10 \mathrm{~mm}$, was present in all solutions; solutions in $B$ also contained $0.1 \mathrm{~mm}$ 4-aminopyridine. Resting potential: $A,-64 \mathrm{mV} ; B$, $-63 \mathrm{mV}$. Scale bars: $10 \mathrm{mV}, 20 \mathrm{msec}$. dicate that repetitive stimulation (even at frequencies as low as $1 \mathrm{~Hz}$ ) selectively reduces the slow components of terminal $\mathrm{Ca}$ current, perhaps by increasing the magnitude of a Ca-activated K current (Lindgren and Moore, 1989). Penner and Dreyer (1986) found that $1 \mu \mathrm{M}$ cadmium blocked only the slow components of $\mathrm{Ca}$ current in mouse motor terminals, but in lizard motor axons $1 \mu \mathrm{M}$ cadmium blocked both fast and slow components of the Ca-dependent afterpotential (Fig. 6B).

In $10 \mathrm{mM}$ TEA the D2 component of the depolarizing afterpotential was usually followed by a $\mathrm{Ca}$-dependent hyperpolarization lasting several seconds, and in $20 \mathrm{mM}$ TEA an even slower, Ca-dependent depolarization (D3) was evident (Morita and Barrett, 1988; unpublished observations). These observations suggest that under our experimental conditions, elevations in intra-axonal [Ca] persisted even longer than D2. Slowly decaying changes in intraterminal [Ca] following tetanic stimulation and/or single stimuli in high-Ca media have been recorded in other nerve terminals injected with Ca-sensitive dyes [squid: Miledi and Parker (1981); Charlton et al. (1982); harnacle photoreceptor: Stockbridge and Ross (1984); Aplysia L10 neuron: Connor et al. (1986); crayfish motor nerve: Delaney et al. (1988)]. This persistence of intraterminal [Ca] might be related to increases in transmitter release observed during repetitive stimulation (e.g., facilitation, potentiation).

\section{Pharmacological classification of Ca channels on lizard motor nerve terminals}

Some neuronal membranes contain multiple types of depolarization-activated Ca channels (reviewed by Miller, 1987). For example, calcium channels in the somatic membrane of cultured dorsal root ganglion neurons have been classified into $\mathrm{L}, \mathrm{N}$, and $T$ subtypes, on the basis of ionic selectivity, single-channel conductance, voltage dependence, and pharmacology (Fox et al., 1987 a, b). Several studies suggest that nerve terminal membranes also contain more than one type of depolarization-activated calcium channel (work on synaptosomes reviewed by Miller, 1987; see also Penner and Dreyer, 1986; Hamilton and Smith, 1987; Lemos and Nowycky, 1989). Calcium entering through $\mathrm{N}$ - and L-type channels is thought to mediate the release of certain neurotransmitters (reviewed by Miller, 1987; see also Hirning et al., 1988; Holz et al., 1988), but some presynaptic
Ca channels do not fit neatly into the $\mathrm{L}, \mathrm{N}$, and $\mathrm{T}$ classification system (Augustine et al., 1987).

Application of the pharmacological criteria used to distinguish $\mathrm{Ca}$ channels on dorsal root ganglion somatic membranes (Fox et al., 1987a, b) suggests that lizard motor terminals contain L-type calcium channels. Both D1 and D2 components were blocked by $1 \mu \mathrm{M}$ cadmium and by GVIA $\omega$-conotoxin (Figs. $6 B$, 9), which block certain $\mathrm{N}$ - and L-type, but not T-type, channels. Also, the depolarizing afterpotential was modified by the dihydropyridines Bay K 8644 and nimodipine (Fig. 10), which arc thought to be selective for L-type channels. Consistent with this finding, Lindgren and Moore (1989) report that $10 \mu \mathrm{M}$ nifedipine (a dihydropyridine Ca channel antagonist) blocked Ca currents recorded extracellularly from TEA-treated lizard motor nerve terminals. Dihydropyridine-sensitive calcium channels can influence transmitter release at the lizard neuromuscular junction since both Lindgren and Moore's and our preliminary findings indicate that $1-10 \mu \mathrm{M}$ Bay K 8644 increases the frequency of spontaneous miniature end-plate potentials at lizard neuromuscular junctions. Bay K 8644 (5 $\mu \mathrm{M})$ also increases spontaneous transmitter release at the mouse neuromuscular junction (Atchison and O'Leary, 1987). Evoked release of ACh at mouse, frog, and lizard neuromuscular junctions is not normally blocked by antagonist dihydropyridines (Atchison and O'Leary, 1987; Hamilton and Smith, 1987; Lindgren and Moore, 1989; and our observations), but this finding does not preclude a role for L-type Ca channels in evoked release because the depolarization associated with the normal action potential may be too brief to allow effective binding of the antagonist dihydropyridines to the motor terminal membrane (Holz et al., 1988). Lindgren and Moore (1989) report that nifedipine does block evoked release at lizard terminals when the action potential is prolonged by TEA.

The voltage dependence of dihydropyridine effects may also explain our finding that nimodipine and Bay K 8644 altered different portions of the $\mathrm{Ca}$-dependent depolarizing afterpotential (Fig. 10). Antagonist dihydropyridines such as nitrendipine, nimodipine, and nifedipine block most effectively at depolarized membrane potentials (Bean, 1984; Sanguinetti and Kass, 1984a; Cohen and McCarthy, 1987; Fox et al., 1987a), consistent with our observation that nimodipine had a greater effect on D1 than 
on the less depolarized D2. In contrast, Bay K 8644 exerts its most potent agonist effects at less depolarized membrane potentials (Hess et al., 1984; Sanguinetti and Kass, 1984b; Fox et al., 1987b), which may explain why Bay K 8644 enhanced D2 but not the more depolarized D1.

Further work is needed to determine whether lizard motor terminals might also contain non-L-type calcium channels and/ or multiple types of $\mathrm{L}$ channels. The $10 \mathrm{~mm}$ TEA we used to make the Ca-dependent potentials large enough to record also depolarized the axonal resting potential by about $12 \mathrm{mV}$ (Barrett et al., 1988), and work in other preparations has shown that steady depolarization inactivates $\mathrm{N}$ - and T-type channels (reviewed by Fox et al., 1987b). The D2 component of the afterpotential appeared at a lower bath [Ca] than the $\mathrm{D} 1$ component (Fig. $4 A$ ), raising the possibility that D1 and D2 might be mediated by different populations of $\mathrm{Ca}$ channels. However, an alternative explanation for this observation is that the axonal/ terminal input resistance might increase with time after the action potential, making a small $\mathrm{Ca}$ influx at the time of D2 easier to detect than a small $\mathrm{Ca}$ influx at the time of D1.

\section{References}

Alvarez-Leefmans, F. J., and R. Miledi (1980) Voltage sensitive calcium entry in frog motoneurones. J. Physiol. (Lond.) 308: 241-257.

Anderson, A. J., and A. L. Harvey (1987) $\omega$-Conotoxin does not block the verapamil-sensitive calcium channels at mouse motor nerve terminals. Neurosci. Lett. 82: 177-180.

Atchison, W. D., and S. M. O'Leary (1987) Bay K 8644 increases release of acetylcholine at the murine neuromuscular junction. Brain Res. 419: 315-319.

Augustine, G. J., M. P. Charlton, and S. J. Smith (1987) Calcium action in synaptic transmitter release. Annu. Rev. Neurosci. 10: 633693.

Baker, M., H. Bostock, P. Grafe, and P. Martius (1987) Function and distribution of three types of rectifying channel in rat spinal root myelinated axons. J. Physiol. (Lond.) 383: 45-67.

Barrett, E. F., and J. N. Barrett (1976) Separation of two voltagesensitive potassium currents, and demonstration of a tetrodotoxinresistant calcium current in frog motoneurones. J. Physiol. (Lond.) 255: 737-774.

Barrett, E. F., and J. N. Barrett (1982) Intracellular recording from vertebrate myelinated axons: Mechanism of the depolarizing afterpotential. J. Physiol. (Lond.) 323: 117-144.

Barrett, E. F., and K. Morita (1987) Calcium-dependent potentials in lizard motor nerve terminals. Soc. Neurosci. Abstr. 13: 312.

Barrell, E. F., K. Morita, and K. A. Scappaticci (1988) Effects of tetraethylammonium on the depolarizing after-potential and passive properties of lizard myelinated axons. J. Physiol. (Lond.) 402: 6578.

Bean, B. P. (1984) Nitrendipine block of cardiac calcium channels: High-affinity binding to the inactivated state. Proc. Natl. Acad. Sci. USA 81: 6388-6392.

Brigant, J. L., and A. Mallart (1982) Presynaptic currents in mouse motor endings. J. Physiol. (Lond.) 333: 619-636.

Charlton, M. P., S. J. Smith, and R. S. Zucker (1982) Role of presynaptic calcium ions and channels in synaptic facilitation and depression at the squid giant synapse. J. Physiol. (Lond.) 323: 173-193.

Cohen, C. J., and R. T. McCarthy (1987) Nimodipine block of calcium channels in rat anterior pituitary cells. J. Physiol. (Lond.) 387: 195225.

Connor, J. A., R. Kretz, and E. Shapiro (1986) Calcium levels measured in a presynaptic neurone of Aplysia under conditions that modulate transmitter release. J. Physiol. (Lond.) 375: 625-642.

Delaney, K. R., D. W. Tank, and R. S. Zucker (1988) Presynaptic calcium at motor nerve terminals during post-tetanic potentiation. Soc. Neurosci. Abstr. 14: 1206.

Feldman, D. H., B. M. Olivera, and D. Yoshikami (1987) Omega conus toxin: A peptide that blocks calcium channels. FEBS Lett. 214: 295-300.
Fox, A. P., M. C. Nowycky, and R. W. Tsien (1987a) Kinetic and pharmacological properties distinguishing three types of calcium currents in chick sensory neurones. J. Physiol. (Lond.) 394: 149-172.

Fox, A. P., M. C. Nowycky, and R. W. Tsien (1987b) Single-channel recordings of three types of calcium channels in chick sensory neurones. J. Physiol. (Lond.) 394: 173-200.

Gordon, T. R., J. D. Kocsis, and S. G. Waxman (1988) Evidence for the presence of two types of potassium channels in the rat optic nerve. Brain Res. 447: 1-9.

Gundersen, C. B., B. Katz, and R. Miledi (1982) The antagonism between botulinum toxin and calcium in motor nerve terminals. Proc. R. Soc. London [Biol.] 216: 369-376.

Hamilton, B. R., and D. O. Smith (1987) Calcium currents in mammalian motor nerve terminals. Soc. Neurosci. Abstr. 13: 312.

Harada, Y., and T. Takahashi (1983) The calcium component of the action potential in spinal motoneurones of the rat. J. Physiol. (Lond.) 335: 89-100.

Hess, P., J. B. Lansman, and R. W. Tsien (1984) Different modes of $\mathrm{Ca}$ channel gating behaviour favoured by dihydropyridine $\mathrm{Ca}$ agonists and antagonists. Nature 311: 538-544.

Hirning, L. D., A. P. Fox, E. W. McCleskey, B. M. Olivera, S. A. Thayer, R. J. Miller, and R. W. Tsien (1988) Dominant role of N-type $\mathrm{Ca}^{2+}$ channels in evoked release of norepinephrine from sympathetic neurons. Science 239: 57-61.

Holz, G. G. IV, K. Dunlap, and R. M. Kream (1988) Characterization of the electrically evoked release of substance $P$ from dorsal root ganglion neurons: Methods and dihydropyridine sensitivity. J. Neurosci. 8: 463-471.

Katz, B. (1969) The Release of Neural Transmitter Substances, Liverpool U. P., Liverpool.

Katz, B., and R. Miledi (1969a) Tetrodotoxin-resistant electric activity in presynaptic terminals. J. Physiol. (Lond.) 203: 459-487.

Katz, B., and R. Miledi (1969b) Spontaneous and evoked activity of motor nerve endings in calcium Ringer. J. Physiol. (Lond.) 203: 689706.

Kerr, L. M., and D. Yoshikami (1984) A venom peptide with a novel presynaptic blocking action. Nature 308: 282-284.

Lemos, J. R., and M. C. Nowycky (1989) Two types of calcium channels coexist in peptide-releasing vertebrate nerve terminals. Neuron 2 : 1419-1426.

Lindgren, C. A., and J. W. Moore (1989) Identification of ionic currents at presynaptic nerve endings of the lizard. J. Physiol. (Lond.) (in press).

Mallart, A. (1984) Presynaptic currents in frog motor endings. Pfluegers Arch. 400: 8-13.

Mallart, A. (1985) A calcium-activated potassium current in motor nerve terminals of the mouse. J. Physiol. (Lond.) 368: 577-591.

Mallart, A. (1986) Some properties of calcium current in mouse motor endings. In Calcium, Neuronal Function and Transmitter Release, R. Rahamimoff and B. Katz, eds., pp. 35-45, Martinus Nijhoff, Boston.

McCleskey, E. W., A. P. Fox, D. Feldman, B. M. Olivera, R. W. Tsien, and D. Yoshikami (1987) $\omega$-Conotoxin: Direct and persistent blockade of specific types of calcium channels in neurons but not muscle. Proc. Natl. Acad. Sci. USA 84: 4327-4331.

Miledi, R., and I. Parker (1981) Calcium transients recorded with arsenazo III in the presynaptic terminal of the squid giant synapse. Proc. R. Soc. London [Biol.] 212: 197-211.

Miller, R. J. (1987) Multiple calcium channels and neuronal function. Science 235: 46-52.

Morita, K., and E. F. Barrett (1988) Lizard motor nerve terminals have two calcium-dependent potassium conductances. Soc. Neurosci. Abstr. 14: 70 .

Obaid, A. L., R. K. Orkand, H. Gainer, and B. M. Salzberg (1985) Active calcium responses recorded optically from nerve terminals of the frog neurohypophysis. J. Gen. Physiol. 85: 481-489.

Penner, R., and F. Dreyer (1986) Two different presynaptic calcium currents in mouse motor nerve terminals. Pfluegers Arch. 406: 190197.

Rahamimoff, R., S. D. Erulkar, A. Lev-Tov, and H. Meiri (1978) Intracellular and extracellular calcium ions in transmitter release at the neuromuscular synapse. Ann. NY Acad. Sci. 307: 583-598.

Ross, W. N., and A. E. Stuart (1978) Voltage-sensitive calcium channels in the presynaptic terminals of a decrementally-conducting photoreceptor. J. Physiol. (Lond.) 274: 173-191. 
Sanguinetti, M. C., and R. S. Kass (1984a) Voltage-dependent block of calcium channel current in the calf cardiac Purkinje fiber by dihydropyridine calcium channel antagonists. Circ. Res. 55: 336-348.

Sanguinetti, M. C., and R. S. Kass (1984b) Regulation of cardiac calcium channel current and contractile activity by the dihydropyridine Bay K 8644 is voltage-dependent. J. Mol. Cell. Cardiol. 16. $667-670$.

Sano, K., K. Enomoto, and T. Maeno (1987) Effects of synthetic $\omega$-conotoxin, a new type $\mathrm{Ca}^{2+}$ antagonist, on frog and mouse neuromuscular transmission. Eur. J. Pharmacol. 141: 235-241.

Silinsky, E. M. (1985) The biophysical pharmacology of calcium-dependent acetylcholine secretion. Pharmacol. Rev. 37: 81-132.
Stockbridge, N., and W. N. Ross (1984) Localized $\mathrm{Ca}^{2+}$ and calciumactivated potassium conductances in terminals of a barnacle photoreceptor. Nature 309: 266-268.

Walrond, J. P., and T. S. Reese (1985) Structure of axon terminals and active zones at synapses on lizard twitch and tonic muscle fibers. J. Neurosci. 5: 1118-1131,

Walton, K., and B. P. Fulton (1986) Ionic mechanisms underlying the firing properties of neonatal motoneurons studied in vitro. Neuroscience 19: 669-683. 